P6 (continued)

Objective: To determine whether maintaining a nutrition blog increased student confidence in developing consumer nutrition messages.

Target Audience: 36 undergraduate and 3 graduate students enrolled in a community nutrition course.

Theory, Prior Research, Rationale: The project was based on the Theory of Planned Behavior. The purpose was to increase students' confidence about communicating nutrition to consumers, thus increasing students' intentions to do so as professionals.

Description: After choosing a nutrition topic, students posted one original blog weekly for 8 weeks. Each student assessed the literacy level on one blog using the SMOG Readability Index and the Flesch - Kincaid Grade Level. At completion of the assignment, students evaluated the blog project using a 10 item, 5-point Likert scale questionnaire. Students reported the change in their self-efficacy to write for low literacy groups and use technology to disseminate nutrition information.

Evaluation: Survey item response differences were evaluated using the Wilcoxon signed-rank test. Median before and after Likert responses were significantly different $(p=.000)$ for 3 items: comfort writing about nutrition for the public; understanding how to use the internet to communicate nutrition to the public; and writing for low literacy audiences. Median responses improved from somewhat agree on all three items to agree and strongly agree on writing.

Conclusions and Implications: Writing nutrition blogs may allow students to develop skills and confidence communicating heath message using technology. Projects based on practical application and utilizing relevant technology may create confident entry level practitioners.

Funding: Supplemental Nutrition Assistance Program.

\section{P7 Comparison of Exam Completion Order to Grade in College Students Enrolled in a Nutrition Science Class}

Elizabeth Hilliard, MS, RD, elizabeth.hilliard@ndsu.edu, North Dakota State University, EML Hall 351, PO Box 6050, Department 2620, Fargo, ND 58104; S. David, PhD, ATC; B. Hill, PhD

Objective: To determine if exam completion order is predictive of exam score and final grade in the class.

Design, Setting and Participants: Four exams (3 -100 points each and 1 - 150 points) were administered to 120 undergraduate students enrolled in an introductory nutrition science course. Students completed exams in class, and the instructor maintained exams in the order in which they were submitted. Exam scores and student ID's were recorded by finish order, and final course grade was also recorded.

Outcome Measures and Analysis: Using SPSS, linear regression assessed whether finish order was predictive of exam score and final grade. Correlation was used to determine if finish order was associated with exam score and final grade.
Results: Finish order did not predict exam score $(\mathrm{p}=.249)$, $\mathrm{R}=0.054$ and $\mathrm{R} 2=0.003$. Exam 2 finish order was a weak, significant predictor of final grade $(\mathrm{p}=.05), \mathrm{R}=.213$ and $\mathrm{R} 2=.045$. Exam 2 and 3 finish orders were positively correlated with final course grade, those finishing later earned higher final grades ( $\mathrm{p}=.027$ and .047 respectively). Finish orders were correlated with each other, meaning students finished consistently around the same time $(r=.721-.833$, $\mathrm{P}<.05$ ).

Conclusions and Implications: Finish order did not predict exam grade. Exam 2 and 3 finish orders were weakly, positively correlated with final course grade. Factors related to this correlation include: better student understanding of exam format and content leading to better student preparation, and more challenging content on exams. Further research should explore student exam preparation versus exam finish order.

Funding: None.

\section{P8 Promoting Cultural Competency in Nutrition Students Through Training Paraprofessionals in an International Setting}

ElizabethWall-Bassett, PhD, RD, wallbassette@ecu.edu, East Carolina University, Mailstop 505, Greenville, NC 27858; N. Harris, MPH, RD, LDN

Objective: An international community-based nutrition education model was developed between US and Caribbean partners to integrate diversity training, cultural competence, and nutrition education through a trainthe-trainer program.

Target Audience: Dietetic students, Caribbean nationals.

Theory, Prior Research, Rationale: Diversity training and cultural competence must become fundamental components of university curricula.

Description: Dietetic students $(n=5)$, university nutrition science faculty $(\mathrm{n}=2)$, and Caribbean nationals $(n=7)$ developed eight culturally specific nutrition education modules on basic nutrition, sanitation and safety, and risk reduction and management of prevalent noncommunicable chronic diseases during spring semester 2009. Nutrition students presented the modules for Community Health Aides $(n=20)$ onsite in the Commonwealth of Dominica during summer 2009.

Evaluation: Students responded to structured prompts pertaining to challenges of course development, personal and community impact of the program, and memorable/learning experiences through reflective journals. Participants completed a satisfaction survey and discussed their opinions in a focus group. Themes of personal growth, improved cultural competence, and skill development emerged. Participants agreed that the workshop was a wonderful avenue to connect amongst their communities, establish collegial partnerships, and work with others from different cultures.

Conclusions and Implications: Nutrition education workshops for Community Health Aides can be extremely useful tools for developing a larger base of knowledge, 


\section{P8 (continued)}

increasing health awareness, and strengthening primary care and community outreach among paraprofessionals in an underserved population. Likewise, this service learning model exposed students to innovative strategies and critical thinking skills necessary to succeed in a dynamic, global profession. Utilizing this educational model will prepare students to be more competent providers and leaders in an increasingly diverse and challenging environment.

Funding: None.

\section{P9 Increasing Learning Potential in Entry Level Nutrition Students Through Online Tutorial}

HeidiWengreen, PhD, RD, heidi.wengreen@usu.edu, Utah State University, 8700 Old Main Hill, Logan, UT 84322; A. Litchford, MS, RD; M. Graff, MS, RD

Objective: To assess whether implementation of an online tutoring program MasteringNutrition $₫$ by Pearson Education produces measurable gains in student learning outcomes.

Target Audience: Students of a general education nutrition course Spring of 2013 (no Mastering; $n=182$ ), Fall of 2013 (Mastering; $\mathrm{n}=86$ ), and Spring of 2014 (Mastering; $\mathrm{n}=410$ ).

Theory: Research conducted by Pearson $₫$ indicates that the inclusion of Mastering $@$ tutoring programs into existing courses has the ability to increase student performance on assessments and total course grades.

Description: NDFS 1020 is an introductory nutrition course taught in a blended style. Course structure includes course lectures (60\% of instruction time) and online assignments (40\% of instruction time), Mastering® is included as part of required assignments. Learning outcome progress was measured by questions on a pre-semester quiz, final exam, and posttest six months after course completion. Assessments measured student progress based on course learning objectives.

Evaluation: Results of statistical tests reported no significant difference in test scores for each group over time. Students who scored lower than the mean on the pretest and used the Mastering $\odot$ program demonstrated greater improvements in final and posttest scores compared to those who scored higher than the mean on the pretest $(\mathrm{p}=<0.001)$.

Conclusions and Implications: Implementation of online tutoring program did not significantly improve overall student outcomes. Online tutorial programs may be helpful for students who are enrolled in courses where they have little prior knowledge.

Funding: None.

\section{P10 Efficacy of Problem-Based Learning in an Integrated Skills Course at Improving Dietetics Students' Clinical Reasoning Skills}

Kathy Stanczyk,PhD, RD, LD, kstanczyk@murraystate.edu, Murray State University, 164 Blissful View Drive, Murray, KY 42071; C. Hruskocy, PhD, Capella University; C. Houston, PhD, RD, LD, CFCS, Fontbonne University; K. Ligeikis, EdD, Capella University
Objective: The purpose of the study was to investigate the efficacy of problem-based learning in an integrated skills course at improving dietetics students' clinical reasoning skills.

Target Audience: Dietetics educators and students enrolled in Didactic Programs in Dietetics (DPD).

Theory: Graduates of Didactic Programs in Dietetics (DPD) have been reported to enter supervised professional practice with deficient clinical reasoning skills. Some researchers have suggested that one factor contributing to deficient clinical reasoning skills is the fragmented course sequencing of a traditional dietetics curriculum. An integrated curriculum and problem-based learning (PBL) are two curricular strategies argued to enhance clinical reasoning skills. Vertical integration involves the application of learning to real-life scenarios, placing context at the forefront of learning. One teaching strategy used to foster vertical integration is PBL. PBL is argued to enhance students' critical thinking skills and clinical problem-solving capabilities.

Description: An integrated skills course utilizing PBL was taught in the final semester of a DPD program. Case studies followed by group discussions were used to address various lifecycle stages, diseases, and skills essential for entry into supervised professional practice.

Evaluation: An objective structured clinical examination (OSCE) covering a variety of lifecycle stages, diseases, and skills was used to assess the clinical reasoning skills of dietetics students who completed the integrated skills course, and their OSCE scores were compared to those of a control group. Conclusions and Implications: Results from this study suggest that incorporation of an integrated skills course near the end of a dietetics curriculum could potentially improve students' clinical reasoning skills and confidence level.

Funding: None.

\section{P11 Assessing the Impact of Organized} Community Service on Students' Attitudes

Krisha Thiagarajah,PhD, RD, kthiagar@indiana.edu, Indiana University, 1025 E. 7th Street, Bloomington, IN 47405

Objective: To examine the impact of organized community service on students' attitudes toward community service.

Target Audience: All participants were enrolled in a community nutrition class (intervention group - 20 students) or health class (control group - 30 students) at a Midwest-university.

Theory, Prior Research, Rationale: Even though community service is becoming an essential component of many courses taught in universities, there is limited research on the impact of service-learning on students.

Description: The intervention class participated in a semester long organized community service project providing nutrition education to children as a class requirement. The control health class didn't participate in any organized community service. 